\title{
A Pleuro-Peritoneal Communication through the Diaphragm affected with Lymphangioleiomyomatosis
}

\author{
Yumiko Takagi ${ }^{1}$, Teruhiko Sato ${ }^{1}$, Yoshiteru Morio ${ }^{1}$, Toshio Kumasaka ${ }^{2}$, Keiko Mitani ${ }^{3}$, \\ Hideaki Miyamoto ${ }^{4}$, Shin-ichiro Iwakami ${ }^{5}$, Yuzo Kodama ${ }^{1}$, Emi Onuma ${ }^{6}$, \\ Kuniaki Seyama ${ }^{1}$ and Kazuhisa Takahashi ${ }^{1}$
}

\begin{abstract}
A 30-year-old Japanese woman with lymphangioleiomyomatosis (LAM) developed a left chylothorax and chylous ascites. A pleuro-peritoneal communication was confirmed by a scintigram with ${ }^{99} \mathrm{mTc}$-labeled macroaggregated-albumin injected into the peritoneal cavity. Video-assisted thoracic surgery revealed a protruding papillary lesion on the left diaphragm. Chyle was oozing into the pleural cavity through this lesion. Histopathological analyses demonstrated that the protrusion was a diaphragmatic LAM lesion and that LAMassociated lymphangiogenesis enabled communication between the pleural and peritoneal cavities through lymphatic vessels. This case demonstrated a new mechanism for chylous pleural effusion in LAM and illustrates the significance of LAM-associated lymphangiogenesis.
\end{abstract}

Key words: lymphangioleiomyomatosis, chylothorax, chylous ascites, pleuro-peritoneal communication, lymphangiogenesis

(Inter Med 49: 439-445, 2010)

(DOI: 10.2169/internalmedicine.49.2714)

\section{Introduction}

Lymphangioleiomyomatosis (LAM) is an uncommon disease that occurs exclusively in women of reproductive ages. This condition is characterized by the infiltration of abnormal smooth muscle-like cells (LAM cells) and the cystic destruction of the lung (1). Clinical manifestations of LAM include progressive dyspnea on exertion, recurrent pneumothoraces, hemoptysis, and cough. Furthermore, extrapulmonary abnormalities, including angiomyolipomas or lymphangioleiomyomas of the axial lymphatics, are present in some patients (2-5).

Chylothorax is a well-known complication of LAM (2-4, 6-8). It usually results from the obstruction or disruption of the thoracic duct or its tributaries (7, 9-11). We herein report a patient who experienced a left chylothorax and chylous ascites at the time of the diagnosis of LAM. The patient underwent video-assisted thoracic surgery and a protruding papillary LAM lesion on the left diaphragm was confirmed to be the conduit of chyle leaking from the peritoneal space into the pleural cavity. The present case indicates a new mechanism for the development of LAMassociated chylothorax and illustrates the significance of LAM-associated lymphangiogenesis in the pathophysiology of LAM.

The histopathological findings of the diaphragm and cytological findings of the ascites of this patient have been in part reported in a previous study as the registered case number of LNF79 (12). However, we consider that a description of this patient's clinical characteristics is valuable in order to elucidate a new mechanism for the simultaneous occurrence of chylous pleural effusion and ascites in LAM patients.

\footnotetext{
${ }^{1}$ Department of Respiratory Medicine, Juntendo University School of Medicine, Tokyo, ${ }^{2}$ Division of Pathology, Japanese Red Cross Medical Center, Tokyo, ${ }^{3}$ Department of Human Pathology, Juntendo University School of Medicine, Tokyo, ${ }^{4}$ Department of General Thoracic Surgery, Southern Tohoku Research Institute \& General Hospital, Koriyama, ${ }^{5}$ Division of Respiratory Medicine, Shizuoka Juntendo University Hospital, Izu-no-kuni and ${ }^{6}$ Department of Respiratory Medicine, Tokyo Metropolitan Koto Geriatric Medical Center, Tokyo

Received for publication July 26, 2009; Accepted for publication November 16, 2009

Correspondence to Dr. Teruhiko Sato, satoteru@juntendo.ac.jp
} 
Table 1. Laboratory Findings of Chylous Pleural Effusion and Ascites

\begin{tabular}{lcccccccc}
\hline & $\begin{array}{c}\text { Total Protein } \\
(\mathrm{g} / \mathrm{dL})\end{array}$ & $\begin{array}{c}\mathrm{LDH} \\
(\mathrm{IU} / \mathrm{mL})\end{array}$ & $\begin{array}{c}\text { Specific } \\
\text { gravity }\end{array}$ & $\begin{array}{c}\text { Cell number } \\
(/ \mu \mathrm{L})\end{array}$ & Fibrin & $\begin{array}{c}\text { T-Cho } \\
(\mathrm{mg} / \mathrm{dL})\end{array}$ & $\begin{array}{c}\text { TG } \\
(\mathrm{mg} / \mathrm{dL})\end{array}$ & $\begin{array}{c}\text { VEGF-D } \\
(\mathrm{pg} / \mathrm{mL})\end{array}$ \\
\hline Pleural effusion & 5.4 & 361 & 1.019 & 14,124 & + & 80 & 1391 & 7,008 \\
\multirow{2}{*}{ Ascites } & 5.8 & 261 & 1.020 & 15,598 & + & 84 & 1226 & 7,129 \\
\hline
\end{tabular}
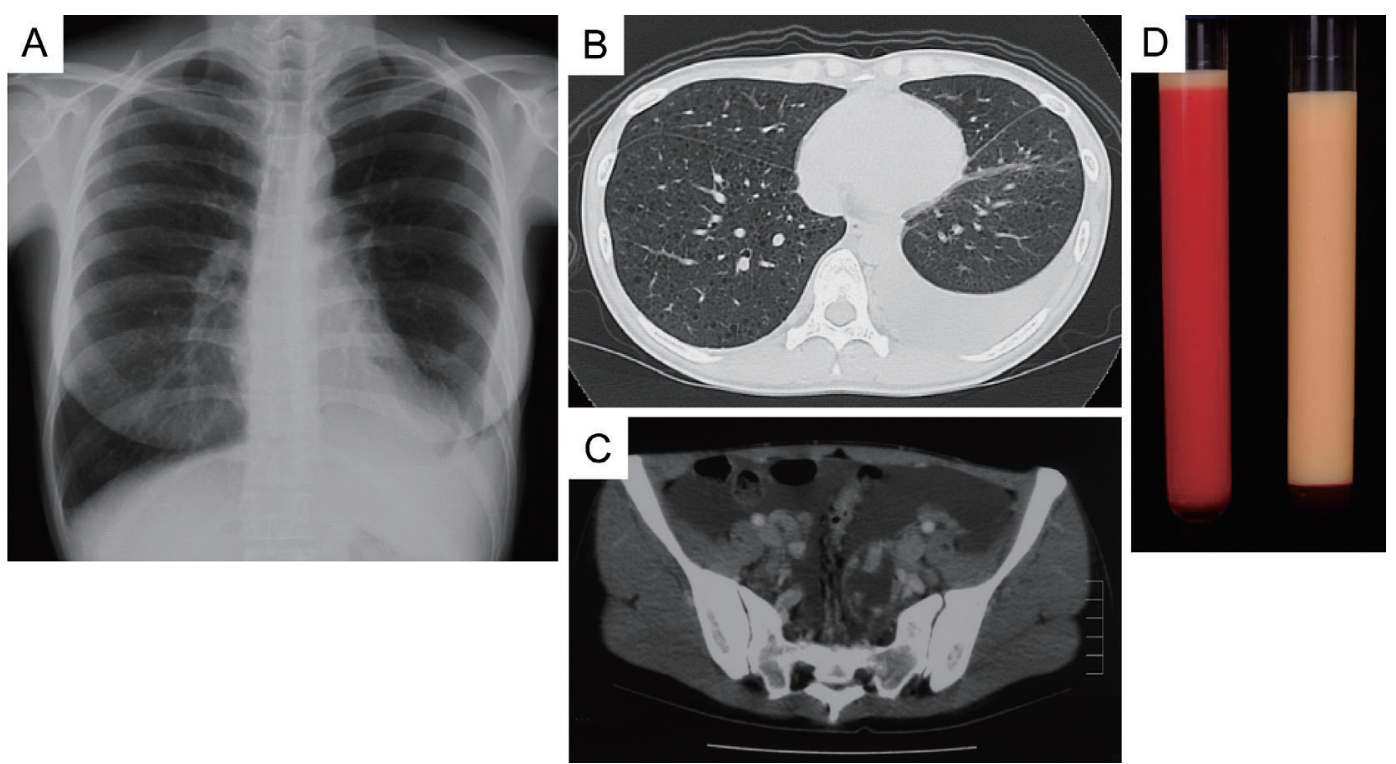

Figure 1. Radiological findings on admission. A: A chest radiograph showed an abnormal density at the left costophrenic angle, which indicated the presence of a pleural effusion. B: Computed tomography (CT) of the chest revealed the left pleural effusion and multiple thin-walled cysts in the both lung fields. C: Contrast-enhanced $\mathrm{CT}$ of the abdomen and the pelvis revealed a moderate amount of ascites and cystic tumors suggestive of lymphangioleiomyomas near the right iliac vein. D: Appearance of the pleural effusion: prior (left) and after (right) low-speed centrifugation.

\section{Case Report}

A 30-year-old woman was admitted to our hospital for an evaluation of a left pleural effusion that had been detected during an annual health checkup at her workplace in May 2003. She worked as a clerk in a hospital and had experienced exertional dyspnea for several months prior to the admission. The physical examination suggested that she had ascites in addition to a pleural effusion and these findings were confirmed by computed tomography (CT) of the chest and abdomen. Because the chest CT also revealed multiple thin-walled cysts scattered through both lung fields, the patient was suspected to be affected with LAM and was referred to our hospital.

On admission, a physical examination revealed the following: height, $157.5 \mathrm{~cm}$; body weight, $45 \mathrm{~kg}$; body temperature, $36.2^{\circ} \mathrm{C}$; blood pressure, $88 / 54 \mathrm{mmHg}$; pulse rate, $72 / \mathrm{min}$; and respiration rate, $18 / \mathrm{min}$. Her respiratory sounds were slightly diminished in the left lower lung field. The abdomen was slightly distended, which indicated the presence of ascites. No cutaneous or neurological abnormalities, suggestive of tuberous sclerosis complex (TSC) were observed. There was no family history of TSC, although she had a past history of ulcerative colitis at 25 years of age. Blood tests showed a white blood cell count of $4,100 / \mu \mathrm{L}$ with $13.8 \%$ lymphocytes, hemoglobin of $12.1 \mathrm{~d} / \mathrm{dL}$, and platelet count of $22.8 \times 10^{4} / \mu \mathrm{L}$, total protein level of $7.3 \mathrm{~g} / \mathrm{dL}$, albumin level of $3.8 \mathrm{~g} / \mathrm{dL}$, total cholesterol level of $130 \mathrm{mg} / \mathrm{dL}$, and triglyceride level of $85 \mathrm{mg} / \mathrm{dL}$. An arterial blood gas analysis under the room air conditions showed a moderate degree of hypoxemia $\left(\mathrm{PaO}_{2} 66.7\right.$ Torr $)$ at rest. The chest $\mathrm{X}$ ray on admission (Fig. 1A) revealed a left pleural effusion with mild to moderate retention, and the chest $\mathrm{CT}$ revealed a moderate accumulation of the left pleural effusion with the multiple thin-walled cysts in the both lung fields (Fig. 1B). These findings were consistent with LAM. The pelvic CT demonstrated ascites and cystic tumorous lesions near the right iliac vein, which was suggestive of lymphangioleiomyoma (Fig. 1C); however, there was no evidence of any renal angiomyolipoma.

Thoracentesis yielded milky-red fluid. Low-speed centrifugation separated the milky turbid fluid from the pellet of red blood cells (Fig. 1D). The fluid was confirmed to be chyle due to the fact that it had a total cholesterol level of $80 \mathrm{mg} / \mathrm{dL}$ and a triglyceride level of $1,391 \mathrm{mg} / \mathrm{dL}$ (Table 1). The ascites obtained by abdominocentesis also consisted of bloody chyle, which possessed an appearance and laboratory 


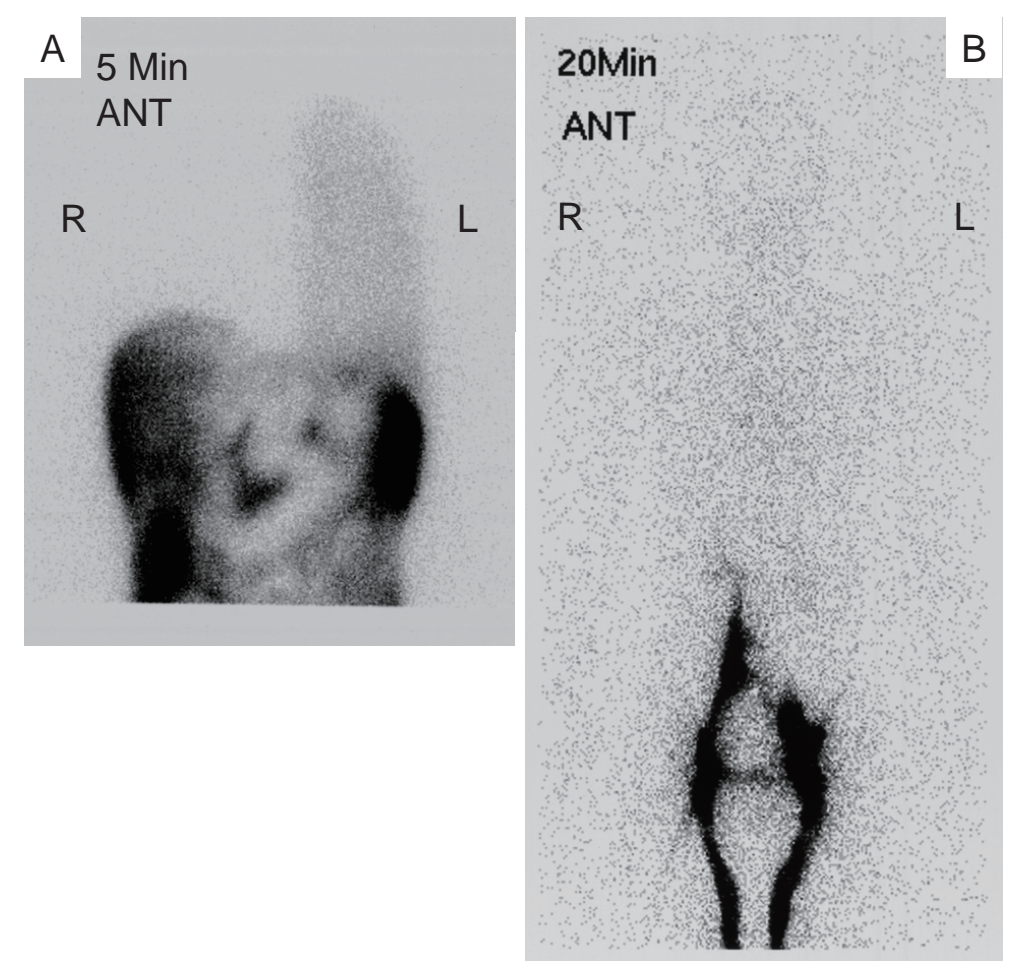

Figure 2. Scintigraphic examinations suggested the communication between the peritoneal and thoracic cavities as well as the obstruction of lymphatic flow. A: ${ }^{99} \mathrm{mTc}$-labeled macroaggregated albumin was injected into the peritoneal cavity with the patient in the supine position. The radioactive signal was detected in accord with the left pleural cavity only 5 minutes after the injection, thus suggesting the existence of a pleuro-peritoneal communication. B: The lymphoscintigraphy using ${ }^{99} \mathrm{mTc}$-labeled human serum albumin (HAS) demonstrated the blockade of lymphatic flow at the pelvic level. The left-sided lymphatic flow was blocked at a lower level than the contralateral flow, in accordance with the existence of the cystic tumor (Fig. 1C). The thoracic duct and axial lymphatics, which should normally be depicted, were not detected. This figure represents the scintigraphy at 20 minutes after the subcutaneous injection of the ${ }^{99} \mathrm{mTc}$-HAS into the bilateral dorsum pedis. The scan was performed from the anterior side of the patient.

findings similar to the pleural effusion (Table 1). Therefore, we postulated that there was a communication between the peritoneal cavity and left pleural cavity. The concentrations of VEGF-D in the pleural effusion and ascites were higher $(7,008$ and $7,129 \mathrm{pg} / \mathrm{mL}$, respectively) than that in serum $(2,861 \mathrm{pg} / \mathrm{mL})$. In order to confirm the pleuro-peritoneal communication, we injected ${ }^{99} \mathrm{mTc}$-labeled macroaggregatedalbumin into the peritoneal cavity of the patient. Radioactive signals were detected in the left pleural cavity only 5 minutes after the injection. The peritoneal cavity was visualized more intensely than the left pleural cavity (Fig. 2A). Lymphoscintigraphy, a scan following subcutaneous injection of ${ }^{99} \mathrm{mTc}$-labeled human serum albumin (HSA) into the dorsum pedis, revealed a lack of depiction of the thoracic duct and axial lymphatics at the level of the pelvis, suggesting an extensive congestion or obstruction of lymphatic flow (Fig. 2B).

Based on the characteristic clinical features, including the fact that the patient was a woman of reproductive age, the presence of multiple thin-walled cysts on the chest CT, and the chylous pleural effusion and ascites, we suspected that the patient was affected with LAM. In order to confirm the diagnosis and to evaluate the lesion on the diaphragm, video-assisted thoracic surgery (VATS) was performed on the 10th hospital day. Macroscopic observation via thoracoscopy revealed milky-red colored chyle and a protruding papillary lesion on the dome of the left diaphragm (Fig. 3A). It was located in the muscular portion near the boundary with the tendinous portion of the left diaphragm. No obvious fenestra was observed on the diaphragm and the chyle appeared to be oozing into the left pleural cavity from the diaphragmatic papillary lesion. This lesion was resected at the base using an automatic stapling device under VATS for the histopathological analyses. An area of erosion was detected on the top of the lesion (Fig. 3A, inset), which was identified as the conduit for chyle to leak from the peritoneal to the pleural cavity. The S8 region of the left lung was also biopsied for the histopathological diagnosis. Microscopic examinations disclosed the proliferation of LAM cells in the lung specimen (Fig. 3B). The protruding papillary lesion was identified as a diaphragmatic LAM lesion where LAM cells had proliferated and destroyed the original diaphragmatic structure including the tendon (Figs. 3C, 3D). Immunohistochemical examination clearly demonstrated that 

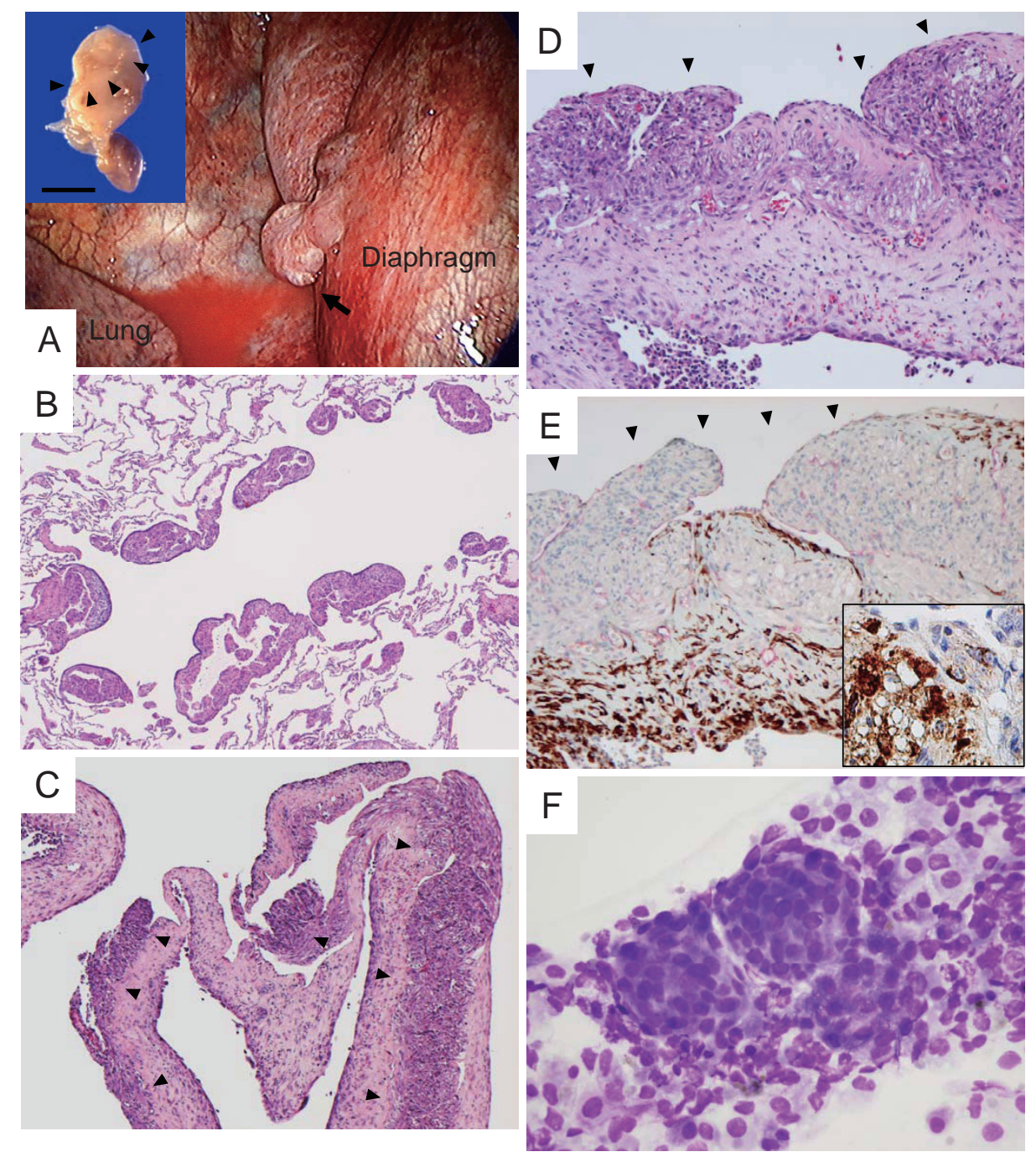

Figure 3. Histopathological findings of the LAM lesions in the lung and the diaphragm. A: Thoracoscopy demonstrated a papillary protrusion (arrow) from the dome of the left diaphragm. The lesion was resected under the video-assisted thoracic surgery (VATS). The inset shows a formalinfixed specimen and the arrowheads indicate an erosion on the top of the protruding lesion (the bar=1 $\mathrm{cm}$ ), which was considered to be the channel for the leakage of chyle from the peritoneal cavity to the pleural cavity. B: Histopathological findings of the lung (Hematoxylin and Eosin staining, with the original magnification $\times 20$ ). Spindle-shaped LAM cells proliferated in nodules in the respiratory bronchiole region. Each LAM nodule had slit-like or dilated spaces, which were considered to be the LAM-associated lymphatic vessels. C-E: Histopathological findings of the papillary protrusion on the left diaphragm. Hematoxylin and Eosin staining (C and D) and double immunostaining (E) with VEGFR-3 (red) and cytokeratin (brown) of the diaphragmatic lesion are presented (the original magnification $\mathrm{C}, \times 200 ; \mathrm{D}$ and $\mathrm{E}, \times 400$ ). Histologically, the resected protruding lesion was identified to be a part of the diaphragm itself that was abnormally thickened and drawn (C and D). The upper and the lower portions of the figures correspond with the pleural and the peritoneal sides, respectively. Proliferating LAM cells (arrowheads) were identified on the pleural side of the diaphragm (C and D). Note that the original structure of the diaphragm, including striated muscle or tendon, was not observed. Immunohistochemical examination $(E)$ clearly demonstrated that the surface of the LAM nodules was not covered with KL1 (cytokeratin)-positive mesothelial cells (brown) and was directly exposed to the pleural cavity (arrowheads). LAM cells were confirmed by HMB45 staining (brown) (the inset in $\mathrm{E}$, the original magnification $\times \mathbf{4 0 0}$ ). The extensive LAM-associated lymphangiogenesis (red) and the proliferating reactive mesothelial cells (brown) in the diaphragm were demonstrated with anti-VEGFR-3 immunostaining. F: LAM cell clusters (LCCs) isolated from the ascites (smear, Diff-Quik stain ${ }^{\mathrm{TM}}$, the original magnification $\times 150$ ). Three clearly demarcated and globular-shaped LCCs, which were surrounded by mesothelial cells and lymphocytes, were observed. 
the thoracic surface of the resected diaphragmatic lesion, especially the top of the protrusive lesion, was not covered with KL1 (cytokeratin)-positive mesothelial cells; furthermore, proliferating LAM cells were directly exposed to the thoracic cavity (Fig. 3E). Antibody against VEGFR-3, a marker of lymphatic endothelial cells, revealed extensive LAM-associated lymphangiogenesis in the LAM-affected diaphragm (Fig. 3E). The abundant LAM-associated lymphatic vessels formed a lymphatic network throughout the diaphragm, thus suggesting that the source of the communication between the pleural and peritoneal cavity was through LAM-associated lymphatic vessels. Numerous LAM cell clusters (LCCs), a globular-shaped cluster of LAM cells enveloped by lymphatic endothelial cells (12), were identified in fluid samples from the ascites (Fig. 3F) and the pleural effusion (not shown). Immediately after the operation, the left pleural effusion disappeared. However, thereafter the patient felt a stronger degree of abdominal distension than before the operation since her abdominal circumference increased approximately $3-5 \mathrm{~cm}$ after the operation. Low-fat diet and as-needed use of diuretics was initiated, but she needed an occasional abdominocentesis to relieve abdominal distension. She was discharged on the 15th post-operative day and thereafter the administration of gonadotropinreleasing analogue (subcutaneous injection of leuprorelin acetate $1.88 \mathrm{mg}$, every 4 weeks) was initiated for the treatment of LAM. She remained free of pleural effusion while she had massive but a tolerable amount of ascites without abdominocentesis. A small amount of left pleural effusion reappeared approximately one year later.

\section{Discussion}

Although chylothorax is one of the major complications of LAM, the incidence of chylous ascites is less common. Moreover, the simultaneous occurrence of chylothorax and chylous ascites is quite rare. Chylothorax has been reported in less than $14 \%$ of LAM patients at presentation and its incidence seems to increase to $22-39 \%$ of patients during the course of disease (2-4, 6-8). In a retrospective study, Ryu et al reported that eight out of 79 patients $(10.1 \%)$ with LAM experienced a chylothorax (10). However, none of these eight patients had chylous ascites during their clinical course, with the exception of one patient who had been diagnosed with chylous ascites 12 years before the onset of the chylothorax (10). Hayashida et al reviewed a total of 173 Japanese LAM patients, including the present patient and found that $21(12 \%)$ patients had a chylothorax and 11 out of 165 patients (7\%) who received abdominal CT or ultrasonography had ascites (2). Regarding this series of patients, only 3 patients (including this patient) simultaneously experienced chylothorax and ascites at the same time (personal communication).

This patient is the first reported case in which a protruding papillary LAM lesion on the left diaphragm was confirmed to be the channel through which chyle leaked from the peritoneal space to the pleural cavity. The putative mechanisms of chylothorax formation in LAM include the following: 1) the leakage of chyle from the thoracic duct or its tributaries as the result of the proximal lymphatic obstruction or direct involvement by LAM, 2) general oozing from pleural lymphatics or collateral vessels, and 3) transdiaphragmatic flow of chylous ascites (10). Porous diaphragm syndrome, an anatomical defect of the diaphragm through which a substance passes from the peritoneal cavity to the pleural space, is a possible explanation of the transdiaphragmatic flow of chyle (13). However, the defect may favour the right side of the diaphragm in this syndrome, and the condition is known to be associated with catamenial pneumothorax (14), hydrothorax complicating peritoneal dialysis $(15,16)$ or Meigs's syndrome $(13,17)$. We ware unable to detect a fenestra on the left diaphragm of the patient under thoracoscopy. We instead directly observed the leakage of chyle through the papillary LAM lesion. Furthermore, the pleural effusion disappeared immediately after the surgical resection of the lesion. Consequently, the transdiaphragmatic flow of chyle from the abdominal cavity through the diaphragmatic lesion was considered to be the etiology of the chylothorax in this patient. Morimoto et al reported that chylorrhea from swollen vessels on the right diaphragm was observed with thoracoscopy in a Japanese LAM patient who had a right chylothorax (18); however, there was no evidence of ascites although an abdominal CT scan revealed iso-density masses in the retroperitoneum that were compatible with lymphangioleiomyomas. Unfortunately, it was unclear in that patient whether the lesion was affected with LAM and LAM-associated lymphangiogenesis since the histopathological evaluation of the swollen vessels on the diaphragm was not conducted.

The human diaphragm has naturally extensive lymphatic networks consisting of stomata, lymphatic vessels, and lacunae (19-22). Two layers of lymphatic vessels are present in the muscular portion of the peritoneal diaphragm while one layer is present in the tendinous portion $(20,22)$. The existence of pleural stomata remains controversial. Li observed round or oval stomata on the diaphragmatic pleura using scanning electron microscopy (23) while Shinohara et al did not (22). Because LCCs travel in the lymphatic stream, a diaphragmatic LAM lesion may develop when LCCs anchor at a specific point inside the lymphatic network in the diaphragm. Alternatively, LCCs in ascites may be implanted onto the diaphragmatic peritoneum through lymphatic stomata, thus causing LAM lesions in the diaphragm. To date, pathological findings of the diaphragmatic LAM lesion are not commonly documented, with the exception of our previous report including the present patient and five autopsy cases (12). In that study, we examined the diaphragms from six patients with LAM, including two patients with a history of chylous pleural effusion, two patients with a history of chylous pleural effusion and ascites, and two patients without a history of chylous effusion. Diaphragmatic LAM lesions were identified in three out of the four patients with a 
history of chylous effusion; however, these lesions were not detected in two autopsy cases without a history of chylous effusion (12). These results suggested the possible pathogenic relationship between chylous effusion and diaphragmatic LAM lesions. Since one patient (LYTK59) (12) had a diaphragmatic LAM lesion only on the pleural side of the left diaphragm, it is plausible that pleural stomata may exist in some patients or develop due to some disease-related condition.

Consistent with the extensive lymphatic involvement in LAM, a lymphangiogenic growth factor, VEGF-D, is expressed by LAM cells and is increased in the serum of LAM patients (24). In this patient, the VEGF-D serum level rose to $2,861 \mathrm{pg} / \mathrm{mL}$; moreover, the VEGF-D levels in pleural effusion and ascites were much higher than that in the serum. The appearance of pleural effusion and ascites in this patient was bloody chyle (Figs. 1D, 3A), suggesting that the chyle was not simply oozing from the normal lymphatic stream or leaking due to the proximal obstruction of lymphatic vessels. Kopfstein et al used an elaborate transgenic mouse model to demonstrate that VEGF-D induced lymphangiogenesis with blood-lymphatic vessel shunts (25). Accordingly, LAM-associated lymphangiogenesis seems to play a central role in LAM-associated chyle leakage, especially in this patient. A surgical ligation of the thoracic duct is often performed in order to control refractory chylous effusion $(3,10)$. However, thoracic duct ligation may fail to control chylothorax or its effect lasted transiently and chylothorax recurred (26). Together with the observation that the prevalence of chylous effusion increased during the course of the disease, these findings may indicate that, not only the lymphatic obstruction or direct involvement by LAM, but also the occurrence of an abnormal communication between the pleural or peritoneal space and the lymphatic circulation through LAM-associated lymphangiogenesis thus play a role in the development of chylothorax and ascites in LAM patients.

\section{Acknowledgement}

This study was supported by Grant-in-Aid for Scientific Research No. 18659242 (Seyama K) and in part by a grant to the Respiratory Failure Research Group from the Ministry of Health, Labour and Welfare, Japan and the High Technology Research Center Grant from the Ministry of Education, Culture, Sports, Science, and Technology, Japan and the Institute for Environmental and Gender-Specific Medicine, Juntendo University, Graduate School of Medicine.

\section{References}

1. Carrington CB, Cugell DW, Gaensler EA, et al. Lymphangioleiomyomatosis. Physiologic-pathologic-radiologic correlations. Am Rev Respir Dis 116: 977-995, 1977.

2. Hayashida M, Seyama K, Inoue Y, Fujimoto K, Kubo K. The epidemiology of lymphangioleiomyomatosis in Japan: A nationwide cross-sectional study of presenting features and prognostic factors. Respirology 12: 523-530, 2007.

3. Johnson SR, Tattersfield AE. Clinical experience of lymphangioleiomyomatosis in the UK. Thorax 55: 1052-1057, 2000.

4. Urban T, Lazor R, Lacronique J, et al. Pulmonary lymphangioleiomyomatosis. A study of 69 patients. Groupe d'Etudes et de Recherche sur les Maladies "Orphelines" Pulmonaires (GERM“O”P). Medicine (Baltimore) 78: 321-337, 1999.

5. Ryu JH, Moss J, Beck GJ, et al. The NHLBI lymphangioleiomyomatosis registry: characteristics of 230 patients at enrollment. Am J Respir Crit Care Med 173: 105-111, 2006.

6. Chu SC, Horiba K, Usuki J, et al. Comprehensive evaluation of 35 patients with lymphangioleiomyomatosis. Chest 115: 10411052, 1999

7. Corrin B, Liebow AA, Friedman PJ. Pulmonary lymphangiomyomatosis. A review. Am J Pathol 79: 348-382, 1975.

8. Taylor JR, Ryu J, Colby TV, Raffin TA. Lymphangioleiomyomatosis. Clinical course in 32 patients. N Engl J Med 323: 1254-1260, 1990.

9. Cornog JL Jr, Enterline HT. Lymphangiomyoma, a benign lesion of chyliferous lymphatics synonymous with lymphangiopericytoma. Cancer 19: 1909-1930, 1966.

10. Ryu JH, Doerr CH, Fisher SD, Olson EJ, Sahn SA. Chylothorax in lymphangioleiomyomatosis. Chest 123: 623-627, 2003.

11. Silverstein EF, Ellis K, Wolff M, Jaretzki A 3rd. Pulmonary lymphangiomyomatosis. Am J Roentgenol Radium Ther Nucl Med 120: 832-850, 1974.

12. Kumasaka T, Seyama K, Mitani K, et al. Lymphangiogenesismediated shedding of LAM cell clusters as a mechanism for dis- semination in lymphangioleiomyomatosis. Am J Surg Pathol 29: 1356-1366, 2005.

13. Kirschner PA. Porous diaphragm syndromes. Chest Surg Clin N Am 8: 449-472, 1998.

14. Kirschner PA. Catamenial pneumothorax: an example of porous diaphragm syndromes. Chest 118: 1519-1520, 2000.

15. Edwards SR, Unger AM. Acute hydrothorax - a new complication of peritoneal dialysis. Jama 199: 853-855, 1967.

16. Nomoto Y, Suga T, Nakajima K, et al. Acute hydrothorax in continuous ambulatory peritoneal dialysis - a collaborative study of 161 centers. Am J Nephrol 9: 363-367, 1989.

17. Saito F, Tashiro H, Honda R, Ohtake H, Katabuchi H. Twisted ovarian tumor causing progressive hemothorax: a case report of porous diaphragm syndrome. Gynecol Obstet Invest 66: 134-137, 2008.

18. Morimoto N, Hirasaki S, Kamei T, et al. Pulmonary lymphangiomyomatosis (LAM) developing chylothorax. Intern Med 39: 738$741,2000$.

19. Li J, Jiang B. A scanning electron microscopic study on threedimensional organization of human diaphragmatic lymphatics. Funct Dev Morphol 3: 129-132, 1993.

20. Li J, Zhao Z, Zhou J, Yu S. A study of the three-dimensional organization of the human diaphragmatic lymphatic lacunae and lymphatic drainage units. Ann Anat 178: 537-544, 1996.

21. Li JC, Yu SM. Study on the ultrastructure of the peritoneal stomata in humans. Acta Anat (Basel) 141: 26-30, 1991.

22. Shinohara H, Kominami R, Taniguchi Y, Yasutaka S. The distribution and morphology of lymphatic vessels on the peritoneal surface of the adult human diaphragm, as revealed by an inkabsorption method. Okajimas Folia Anat Jpn 79: 175-183, 2003.

23. Li J. Ultrastructural study on the pleural stomata in human. Funct Dev Morphol 3: 277-280, 1993.

24. Seyama K, Kumasaka T, Souma S, et al. Vascular endothelial growth factor-D is increased in serum of patients with lymphan- 
Inter Med 49: 439-445, 2010 DOI: 10.2169/internalmedicine.49.2714

gioleiomyomatosis. Lymphat Res Biol 4: 143-152, 2006.

25. Kopfstein L, Veikkola T, Djonov VG, et al. Distinct roles of vascular endothelial growth factor-D in lymphangiogenesis and metastasis. Am J Pathol 170: 1348-1361, 2007.
26. Wolff M. Lymphangiomyoma: clinicopathologic study and ultrastructural confirmation of its histogenesis. Cancer 31: 988-1007, 1973.

(C) 2010 The Japanese Society of Internal Medicine http://www.naika.or.jp/imindex.html 